\title{
Valproate? Lithium comes first ${ }^{\dagger}$ COMMENTARY ON... COCHRANE CORNER
}

\author{
Mary Jane Attenburrow \& Katharine Smith
}

\begin{abstract}
SUMMARY
Maintenance drug treatment in bipolar disorder is a key component of management. There is clinical uncertainty about the relative efficacy of valproate, either alone or in combination, particularly in comparison to lithium. This month's Cochrane corner review aimed to summarise the best evidence available. This commentary puts the findings into their clinical context and updates them in the light of subsequent analyses and the recent revision of the NICE guidelines on bipolar disorder.

DECLARATION OF INTEREST

None
\end{abstract}

This month's Cochrane Corner (Cipriani 2013) is the second update of a review originally published by Macritchie et al in 2001. It had a broad remit: to investigate the effectiveness of valproate (all formulations) in the maintenance treatment of bipolar disorder. As part of this, the review aimed to address the key clinical question of whether valproate or lithium should be used as first-line therapy in the prevention of recurrence. The conclusions from the present review provided key evidence to support the changes made by the National Institute for Health and Care Excellence in its updated guidelines on the treatment of bipolar disorder (NICE 2014), which previously (NICE 2006) recommended valproate as one of the first-line options and which now recommend that lithium alone be offered as the first-line pharmacological treatment.

\section{Clinical setting}

Bipolar disorder is a chronic condition characterised by recurrent mood episodes with significant morbidity and mortality (Kupfer 2005). Maintenance treatment aimed at mood stabilisation (i.e. preventing or attenuating mood episodes) is a key component of management. Drug treatments are the most effective option; psychological therapies (particularly those aimed at psychoeducation) can be effective as an adjunct (Geddes 2013).

\section{Choice of drug treatment in bipolar disorder}

Lithium was the standard maintenance treatment until the 1990s, when valproate became available.
Valproate was licensed by the US Food and Drug Administration (FDA) in 1995. It gained in popularity, particularly in the USA (Blanco 2002), possibly because of its perceived greater tolerability and convenience of use over lithium. Although less widely used in the UK, it is frequently prescribed in routine clinical practice (Hayes 2011; Geddes 2013). Over the past decade, the range of drug treatment options for maintenance has expanded to include atypical antipsychotics.

At the time of Cipriani et al's 2013 Cochrane review, the NICE guidance from 2006 recommended that clinicians should choose between lithium, olanzapine or valproate as monotherapy for maintenance treatment. The Canadian Network for Mood and Anxiety Treatments (CANMAT) guidelines (Yatham 2013) also include lamotrigine, quetiapine, long-acting risperidone and aripiprazole as choices, with the clinician balancing efficacy, tolerability and acceptability. All of these drugs have well-recognised side-effect profiles. In addition, NICE (2014) recommends that valproate should not be prescribed routinely for women of child-bearing potential.

\section{Results of the review}

The review's findings were limited by the small number of trials that could be included. This is not surprising, as such trials, particularly head-to-head comparisons, are challenging to fund and carry out. Overall, the review could not make a confident conclusion about the relative efficacy of valproate versus lithium for maintenance treatment in bipolar disorder. There was some evidence of superiority of valproate over placebo in preventing study withdrawal due to any mood episode $(\mathrm{RR}=0.68,95 \%$ CI $0.49-0.93 ; P=0.02, \mathrm{NNTB}^{\mathrm{a}}=8,95 \%$ CI $5-50 ; 2$ randomised controlled trials (RCTs), 312 participants), but this was less robust than comparable evidence for lithium versus placebo (Burgess 2001).

There was evidence for the superiority of valproate-lithium combination over lithium, but this came from the only trial to have studied these options head to head (Geddes 2010), where the combination was superior to valproate alone in preventing study withdrawal due to episodes of mood disorder $(\mathrm{RR}=0.78,95 \%$ CI $0.63-0.96$, $P=0.02 ; \mathrm{NNTB}=7,95 \%$ CI 4-34; 1 RCT, 220

\section{ROUND THE} CORNER
Mary Jane Attenburrow is Clinical Lead at the NIHR Oxford cognitive health Clinical Research Facility, a Senior Research Fellow at Oxford University Department of Psychiatry and an honorary consultant psychiatrist. Katharine Smith is an honorary consultant psychiatrist at the National Institute for Health Research (NIHR) Oxford cognitive health Clinical Research Facility and Oxford University Department of Psychiatry. She has dual accreditation as a consultant in both general adult and old age psychiatry.

Correspondence Dr Mary Jane Attenburrow, Department of Psychiatry, Warneford Hospital, Oxford OX3 7JX, UK. Email: maryjane.attenburrow@psych.ox.ac.uk

${ }^{\dagger}$ See p. 2, this issue.

a. Number needed to treat to benefit (NNTB) is an estimate of how many people need to receive a treatment before one person would experience a beneficial outcome. For example, if you need to give a stroke prevention drug to 20 people before one stroke is prevented, then the NNTB for that drug is 20. (After Cochrane Collaboration glossary: http://www. cochrane.org/glossary) 
participants). Other important clinical issues, such as health-related quality of life, ability to return to work and attitudes towards treatment, were not addressed in the review because these outcomes were not reported consistently in the primary studies.

The data suggested that valproate may be better tolerated than lithium. However, lithium levels in the trials analysed were somewhat higher than those routinely used in clinical practice (i.e. two of the four studies required a minimum lithium plasma level of $0.8 \mathrm{mmol} / \mathrm{L})$, which may have affected results, as informed management of lithium plasma levels can improve the tolerability and outcome of taking lithium (Mahli 2012).

The common clinical challenges of 'rapid cycling' and non-response to mood-stabilising medication could not be addressed as the numbers extracted from the eligible studies were too small to allow statistical analysis.

\section{Guidelines and evidence since the review was completed}

The recently revised NICE guidelines (NICE 2014) recommend lithium as the first-line option for maintenance treatment. Valproate is no longer recommended as first line, but as a second-line treatment if lithium is poorly tolerated/not suitable and as an adjunct if lithium alone is ineffective. Other second-line treatments suggested include olanzapine and quetiapine.

A recent network meta-analysis by Miura and colleagues (Miura 2014) compared all data (both direct and indirect from a total of 6846 participants) for all pharmacological agents for maintenance treatment in bipolar disorder. Although both valproate and the combination of valproate plus lithium were more effective than placebo in preventing relapse $(\mathrm{RR}=0.63,95 \%$ CI $0.47-0.83$; $\mathrm{RR}=0.52,95 \%$ CI $0.35-0.77$ respectively), neither was more effective than lithium alone $(\mathrm{RR}=0.98$, 95\% CI $0.77-1.28 ; \mathrm{RR}=0.84,95 \%$ CI $0.58-1.21$ respectively).

\section{Conclusions}

This review (Cipriani 2013) carefully assessed the evidence for the use of valproate, an established and frequently used maintenance treatment in bipolar disorder. The results showed that there is in fact limited evidence to support the efficacy of valproate. This, taken together with more recent analyses of the available evidence, should reduce clinical uncertainty when deciding which long-term treatment to use as first line, as the evidence is more convincing for lithium. However, lithium is not always effective, and tolerability and acceptability can be a problem. Under these circumstances, the available evidence supports the use of valproate, either alone or in combination, as a second-line treatment.

\section{Acknowledgements}

The authors acknowledge support from the NIHR Oxford cognitive health Clinical Research Facility and thank John Geddes, Professor of Epidemiological Psychiatry and Andrea Cipriani, Associate Professor at Oxford University Department of Psychiatry for their comments and suggestions. The views expressed are those of the authors and not necessarily those of the NHS, the NIHR or the Department of Health.

\section{References}

Blanco C, Laje G, Olfson M, et al (2002) Trends in the treatment of bipolar disorder by outpatient psychiatrists. American Journal of Psychiatry, 159: 1005-10.

Burgess S, Geddes J, Hawton K, et al (2001) Lithium for maintenance treatment of mood disorders. Cochrane Database of Systematic Reviews, 3: CD003013

Cipriani A, Reid K, Young AH, et al (2013) Valproic acid, valproate and divalproex in the maintenance treatment of bipolar disorder. Cochrane Database of Systematic Reviews, 10: CD003196

Geddes JR, Goodwin GM, Rendell J, et al (2010) Lithium plus valproate combination therapy versus monotherapy for relapse prevention in bipolar I disorder (BALANCE): a randomised open-label trial. Lancet, 375: 385-95.

Geddes JR, Miklowitz DJ (2013) Treatment of bipolar disorder. Lancet, 381: 1672-82.

Hayes J, Prah P, Nazareth I, et al (2011) Prescribing trends in bipolar disorder: cohort study in the United Kingdom THIN primary care database 1995-2009. PLoS One, 6: e28725.

Kupfer D J (2005) The increasing medical burden in bipolar disorder. JAMA, 293: 2528-30.

Valproic acid, valproate and divalproex in the maintenance treatment of bipolar disorder.

Macritchie K, Geddes JR, Scott J, et al (2001) Valproic acid, valproate and divalproex in the maintenance treatment of bipolar disorder. Cochrane Database of Systematic Reviews, 3: CD003196.

Malhi GS, Tanious M, Das P, et al (2012) The science and practice of lithium therapy. Australian and New Zealand Journal of Psychiatry, 46: 192-211.

Miura T, Noma H, Furukawa TA, et al (2014) Comparative efficacy and tolerability of pharmacological treatments in the maintenance treatment of bipolar disorder: a systematic review and network meta-analysis. Lancet Psychiatry, 1: 351-9

National Institute for Health and Care Excellence (2006) Bipolar Disorder: The Management of Bipolar Disorder in Adults, Children and Adolescents, in Primary and Secondary Care (NICE Clinical Guideline 38). NICE.

National Institute for Health and Care Excellence (2014) Bipolar Disorder: The Assessment and Management of Bipolar Disorder in Adults, Children and Young People in Primary and Secondary Care (NICE Clinical Guideline 185). NICE.

Yatham LN, Kennedy SH, Parikh SV, et al (2013) Canadian Network for Mood and Anxiety Treatments (CANMAT) and International Society for Bipolar Disorders (ISBD) collaborative update of CANMAT guidelines for the management of patients with bipolar disorder: update 2013. Bipolar Disorders, 15: 1-44. 